\title{
Viral Shedding Time of SARS-CoV-2 and the Factors Involved: A Retrospective Observational Study
}

\section{Guixian Wu}

Taizhou Hospital of Zhejiang Province,Zhejian University Enze Hospital,Taizhou Enze Mesical Center(Group) ,Department of Respiratory and Critical Care Medicine,Taizhou ,Zhejiang Province, 318050 Ling Lin

Taizhou Hospital of Zhejiang Province,Zhejian University Enze Hospital,Taizhou Enze Mesical Center(Group) ,Department of Respiratory and Critical Care Medicine,Taizhou ,Zhejiang Province, 318050

\section{Susu He}

Taizhou Hospital of Zhejiang Province,Zhejian University Department of Respiratory and Critical Care Medicine,Taizhou ,Zhejiang Province, 317000

\section{Qian Chen}

Taizhou Hospital of Zhejiang Province,Zhejian University Enze Hospital,Taizhou Enze Mesical Center(Group) ,Department of Respiratory and Critical Care Medicine,Taizhou ,Zhejiang Province, 318050

\section{Xiaomai Wu}

Taizhou Hospital of Zhejiang Province,Zhejian University Enze Hospital,Taizhou Enze Mesical Center(Group) ,Department of Respiratory and Critical Care Medicine,Taizhou ,Zhejiang Province, 318050

\section{Shuangquan Yan}

Taizhou Hospital of Zhejiang Province,Zhejian University Enze Hospital,Taizhou Enze Mesical Center(Group) ,Department of Respiratory and Critical Care Medicine,Taizhou ,Zhejiang Province, 318050

\section{Yongpo Jiang}

Taizhou Hospital of Zhejiang Province,Zhejian University,Taizhou ,Zhejiang Province,ICU, Taizhou ,Zhejiang Province, 317000

\section{Weijia Pan}

Taizhou Hospital of Zhejiang Province,Zhejian University Enze Hospital,Taizhou Enze Mesical Center(Group) ,Department of Respiratory and Critical Care Medicine,Taizhou ,Zhejiang Province, 318050 Dongqin Lv ( $\sim$ Ivdq@enzemed.com )

Taizhou Hospital of Zhejiang Province,Zhejian University Enze Hospital,Taizhou Enze Mesical Center(Group) ,Department of Respiratory and Critical Care Medicine,Taizhou ,Zhejiang Province, 318050

\section{Research Article}

Keywords: viral shedding time, risk factors, SARS-CoV-2, COVID-19, digestive tract,respiratory tract,antibodies 
Posted Date: February 3rd, 2021

DOI: https://doi.org/10.21203/rs.3.rs-154418/v1

License: (c) (i) This work is licensed under a Creative Commons Attribution 4.0 International License. Read Full License 


\section{Viral Shedding time of SARS-CoV-2 and the factors involved: A retrospective observational study}

Guixian-Wu ${ }^{1}$ Ling-Lin ${ }^{1}$,Susu-He ${ }^{2}$,Qian-Chen ${ }^{1}$,Xiaomai-Wu ${ }^{1}$,Shuangquan-Yan ${ }^{1}$,Yongpo-Ji ang $^{3}$,Weijia-Pan ${ }^{1}$,Dongqin-Lv ${ }^{1^{*}}$

${ }^{1}$ Taizhou Hospital of Zhejiang Province,Zhejian University Enze Hospital,Taizhou Enze Mesical Center(Group) ,Department of Respiratory and Critical Care Medicine,Taizhou ,Zhejiang Province, 318050

${ }^{2}$ Taizhou Hospital of Zhejiang Province,Zhejian University Department of Respiratory and Critical Care Medicine,Taizhou ,Zhejiang Province, 317000

${ }^{3}$ Taizhou Hospital of Zhejiang Province,Zhejian University,Taizhou ,Zhejiang Province,ICU, Taizhou ,Zhejiang Province, 317000

\#Corresponding Author: Dongqin-Lv Email: Ivdq@enzemed.com

No. 1, East Tongyang Road, Enze Hospital, Luqiao District, Taizhou City, Zhejiang Province 318050 


\begin{abstract}
Background: In December 2019, the discovery of the novel coronavirus was first reported in Wuhan, China, which subsequently and rapidly spread throughout the country and worldwide, resulting in a pandemic.After a year of intense research, our knowledge of the new coronaviruses has gradually improved; however, knowledge regarding the time of their complete clearance from the body and the factors influencing clearance are currently inadequate.

Results:We conducted a retrospective observational study comprising 135 patients above the age of 18 years with a confirmed diagnosis of COVID-19 pneumonia, who were admitted to the Public Health Center of Taizhou Hospital of Zhejiang Province, Zhejiang University from January 23, 2020, to March 11, 2020. The findings regarding the duration of the infection from the time of onset to the time of being asymptomatic (whichever was observed first) indicated that novel coronaviruses were cleared from the respiratory tract in a maximum of 84 days and a minimum of 1 day with a median clearance time (quartile) of $20(13,30)$ days. Moreover, viruses were cleared from the digestive tract in a maximum of 72 days and a minimum of 5 days with a median clearance time (quartile) of $25(20.75,31)$ days. The viral shedding time of SARS in the digestive tract was found to be longer than that in the respiratory tract $(p=0.03)$. Severe disease $(P<0.001)$, advanced age $(P<$ 0.001), lymphopenia $(P=0.01)$ and elevated CRP $(P=0.036)$ were significantly associated with longer clearance time in the respiratory tract. Gender $(P=0.754)$, novel coronavirus antibodies $(P=0.75)$, and antibiotic use $(P=0.093)$ were not associated with the time span required for the novel coronavirus to be cleared from the respiratory tract.

Conclusions: Independent risk factors for the longer clearance time of novel coronaviruses in the digestive tract versus that in the respiratory tract were compared. Severe disease, advanced age, lymphopenia, and elevated CRP were determined to be factors prolonging the clearance of novel coronaviruses.
\end{abstract}

Keywords: viral shedding time, risk factors, SARS-CoV-2, COVID-19, digestive tract, respiratory tract, antibodies 


\section{Background}

In December 2019, the discovery of the novel coronavirus was first reported in Wuhan, China, which subsequently and rapidly spread throughout the country and worldwide, resulting in a pandemic[1-3]. Individuals in over 250 countries and regions have been affected; especially, the cases in the United States, India, Brazil, Russia, and France, have been the most catastrophic. The domestic situation is under control as the outbreak has been effectively managed with a lowering of the geographic temperature; however, the current global situation does not appear optimistic. With a concomitant increase in studies related to the understanding of the novel coronavirus and the implementation of effective prevention and control measures, palliative care can be provided to affected patients. Additionally, mortality rates have substantially decreased, and after a year of intense research, our knowledge of the new coronaviruses has gradually improved; however, knowledge regarding the time of their complete clearance from the body and the factors influencing clearance are currently inadequate. It has been established that novel coronaviruses can be transmitted not only through the respiratory tract but also through the digestive tract[2, 4, 5], urine, blood, and other routes[6], especially in the respiratory tract, with the highest detection rate in the digestive tract[7]. High viral loads have been detected in samples from the respiratory tract, especially in sputum samples.The SARS-COV-2 is mainly transmitted through respiratory droplets,whereas in the digestive system,the transmission is mainly through the fecal-oral route[7, 8].

\section{Methods}

We collected the clinical and laboratory data from 143 patients with confirmed SARS-CoV-2, who were diagnosed with COVID-19 pneumonia between January 23, 2020, to March 11, 2020, based on reverse transcription-polymerase chain reaction (RT-PCR) analysis of respiratory samples. We excluded one pregnant woman, two patients younger than 18 years of age, and five patients whose viral clearance time exceeded 100 days. A total of 135 patients were included in our retrospective study (Figure 1). We followed up these 135 patients with a confirmed diagnosis of COVID-19 pneumonia for 8 months. We defined the time to complete clearance in 
vivo, i.e., from the onset of symptoms to the point when negative results were obtained in the nucleic acid testing of respiratory swabs (sputum, throat swabs, and nasopharyngeal samples) and alimentary-tract samples (feces) and remained negative until follow-up. All methods were carried out in accordance with relevant guidelines and regulations.The diagnosis and severity of the condition on admission and therapeutic regimen were assessed based on the latest SARS-CoV-2 infection guidelines issued by the World Health Organization on March 13, 2020. Patients were categorized into the five following classes depending on disease severity: mild illness, pneumonia, severe pneumonia, acute respiratory distress syndrome (ARDS), and sepsis/septic shock. To simplify the process, mild disease and patients who were asymptomatic carriers were combined as "mild disease;" those with severe pneumonia, sepsis/septic shock were considered to have "severe disease" for the purpose of this study. Thirty asymptomatic patients, who were carriers, were included in this study. Most were diagnosed with pneumonia except for three mildly affected patients, whose imaging data showed no remarkable changes. Patient characteristics, treatment regimens, and outcome data were obtained from their electronic medical records. Informed consent was obtained from patients or their family members; Ethical approval was granted by the Ethics Committee /Institutional Review boardof Enze Hospital of Taizhou ENze Medical (CenterNO.K20200204).

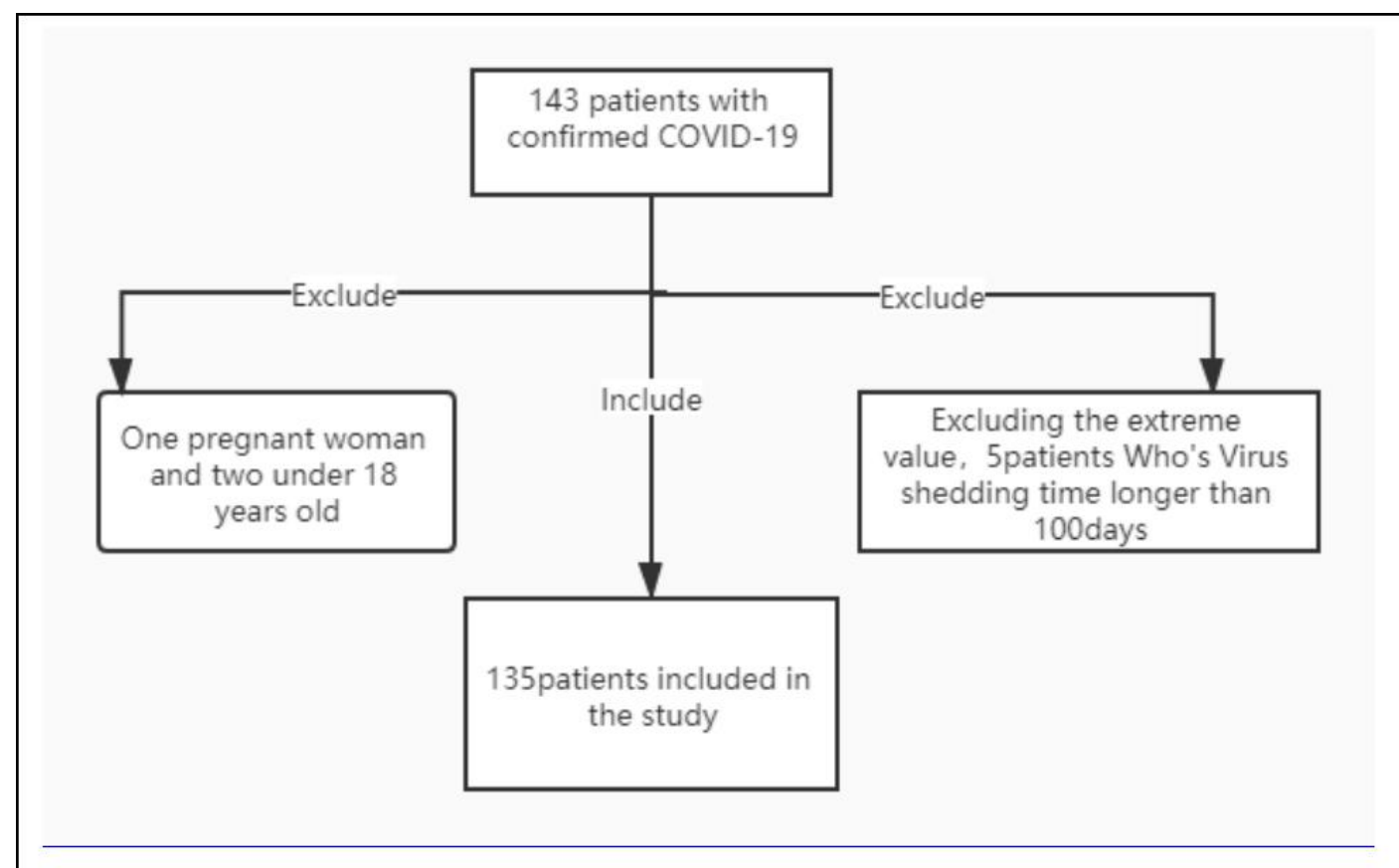

Figure 1. Flow diagram of patients with confirmed COVID-19 included in this study

The diagnosis of SARS-CoV-2 infection was confirmed in all patients using RT-PCR analysis of the respiratory samples, including nasal swabs and digestive samples that were collected daily. The recommended protocols were followed for specimen collection. The diagnosis was based on the criteria recommended by the National Institute for Viral Disease Control and Prevention (China) 
(http://ivdc.chinacdc.cn/kyjz/202001/t20200121_211337.html).

\section{Statistical analysis}

Data were analyzed using SPSS software and continuous variables are expressed as mean \pm standard deviation, or median and interquartile range, as appropriate. Differences between groups were analyzed using $t$-tests or Mann Whitney $\mathrm{U}$ test; categorical variables are expressed using frequencies (percentages) and were analyzed based on the intergroup differences using Chi-square test or Fisher's exact test. Rank correlation analysis was performed on laboratory data to identify the independent risk factors associated with prolonged disease duration after SARS-CoV-2 shedding. Kaplan-Meier survival analysis was used to determine the cumulative negative rate of SARS-CoV-2 viral shedding time and stratified log-rank statistics were used to compare the difference in SARS-CoV-2 clearance between different groups. All statistical analyses were performed using SAS 9.4 software (SAS Institute Inc., Cary, NC, USA). P $<0.05$ was considered statistically significant.

\section{Results}

\section{Patient characteristics}

Table 1 summarizes the baseline characteristics of patients enrolled in this analysis. A statistical description of all baseline characteristics is presented with categorical variables expressed as the number of cases (constituent ratio), normally distributed continuous variables as the mean \pm standard deviation, and non-normally distributed continuous variables as the median (interquartile range). It can be seen in Table 1 that the study cohort comprised 41 (30.4\%) patients with severe infection; 71 (52.6\%) were male, and the number of male and female patients was almost similar with a mean age of $48.3 \pm 13.5$ years. Moreover, $9.6 \%$ of patients had a history of smoking; the main symptoms exhibited by patients included cough (82.2\%), expectoration (38.5\%), fever (78.5\%), whereas some patients complained of fatigue (31. 9\%), chest tightness (31.8\%), nausea (11.1\%), and diarrhea (23.0\%). There were $40.7 \%$ patients with combined underlying diseases, mainly hypertension and 
diabetes mellitus, which accounted for 14.8 and 10.4\%, respectively. Therapeutically, antiviral therapy was provided to all patients and oxygen therapy was used in $67.4 \%$ of patients. Lopinavir, ritonavir, and interferon-alpha were the most commonly used antiviral regimens; For some of the persistently positive patients, the antiviral, abiraterol, was used according to the guidelines.

Table 1: The Demographic datas, Clinical Manifestations and Treatment of the patients were included in the study

\begin{tabular}{|c|c|}
\hline & ALL $(n=140)$ \\
\hline Severe & $41(30.4 \%)$ \\
\hline Age, y & $48.3 \pm 13.5$ \\
\hline Male sex & $71(52.6 \%)$ \\
\hline Current smoker & $13(9.6 \%)$ \\
\hline $\begin{array}{l}\text { Exposure history in } \\
\text { Epidemic Area }\end{array}$ & $67(49.6 \%)$ \\
\hline Exposure history to confirmed & $65(48.1 \%)$ \\
\hline patients, $\%$ (n) & \\
\hline Symptoms & \\
\hline Cough & $111(82.2 \%)$ \\
\hline Expectoration & $52(38.5 \%)$ \\
\hline Fever & $106(78.5 \%)$ \\
\hline Diarrhea & $31(23.0 \%)$ \\
\hline Nausea & $15(11.1 \%)$ \\
\hline hypodynamic & $43(31.9 \%)$ \\
\hline $\begin{array}{l}\text { Chest distress and shortness } \\
\text { of breath }\end{array}$ & $43(31.8 \%)$ \\
\hline Comorbidity, \% (n) & $55(40.7 \%)$ \\
\hline Hypertension & $20(14.8 \%)$ \\
\hline Diabetes & $14(10.4 \%)$ \\
\hline Coronary heart disease & $2(1.5 \%)$ \\
\hline $\begin{array}{l}\text { Duration from illness onset } \\
\text { to hospital admission, } \\
\text { median (IQR), days } \\
\text { Treatment }\end{array}$ & $2(1,5)$ \\
\hline Corticosteroid & $44(32.6 \%)$ \\
\hline Immune Globulin & $39(28.9 \%)$ \\
\hline $\begin{array}{l}\text { Oxygen and High Frequency } \\
\text { Oxygen }\end{array}$ & $91(67.4 \%)$ \\
\hline
\end{tabular}




\begin{tabular}{ll} 
Antibiotics & $55(40.7 \%)$ \\
\hline Antibody & 106 \\
Antibody positive & $93(87.7 \%)$ \\
\multicolumn{2}{c}{ Data are presented as mean \pm standard deviation or $\mathrm{n}(\%)}$.
\end{tabular}

Except for the use of invasive therapy using a ventilator in a critically ill patient, the other critically ill patients received therapy involving frequency oxygen inhalation. Moreover, $32.6 \%$ of the patients were started on hormonal anti-inflammatory prophylaxis to prevent the occurrence of a cytokine storm; $28.9 \%$ of the patients received immunoglobulins and $40.7 \%$ of the patients were prescribed concurrent antibiotics. Eventually, all patients who recovered were discharged. During the follow-up after 8 months, all patients exhibited complete viral transformation and showed no further recurrence. We performed an antibody test in 106 patients and found that 93 of positive patients (87.7\%) and the SARS-COV-2 antibody negative patients were continuously negative for antibodies at the 8-month follow-up; however, those with SARS-COV-2 RNA could still show negative results. Additionally, two of the patients who initially tested positive for SARS-COV-2 antibodies subsequently tested negative at the most recent follow-up, indicating that antibodies cannot persist over a prolonged duration. 
Figure 2: Viral Shedding/Clearance Time of SARS-COV-2 in Digiestion and Respitory

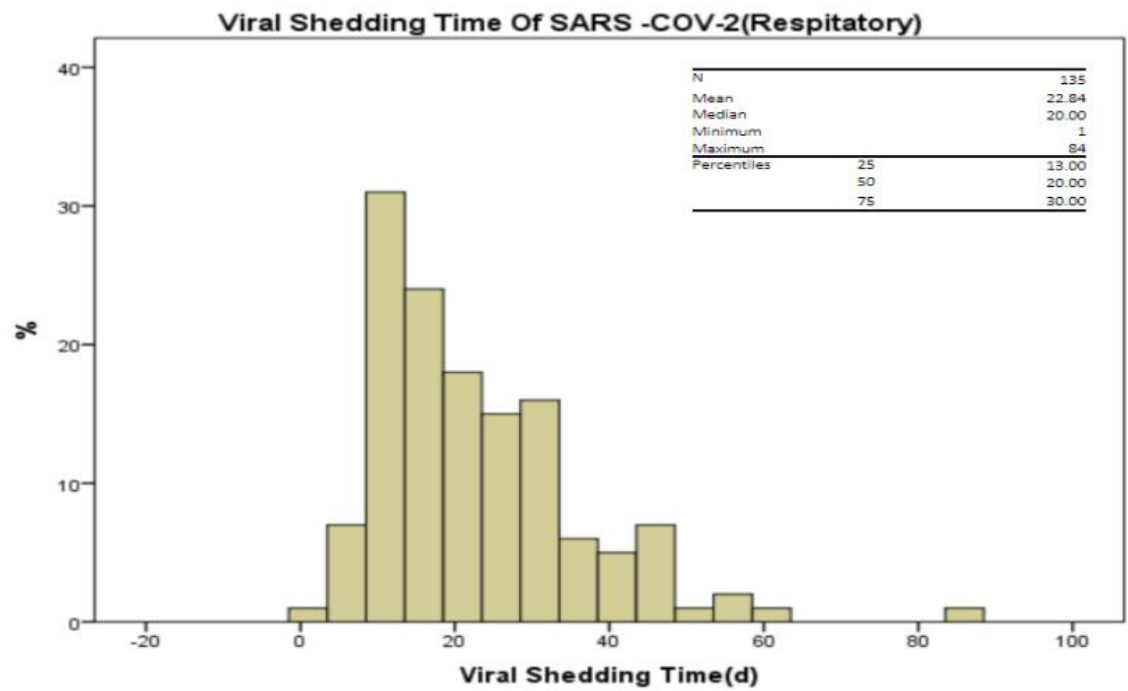

A

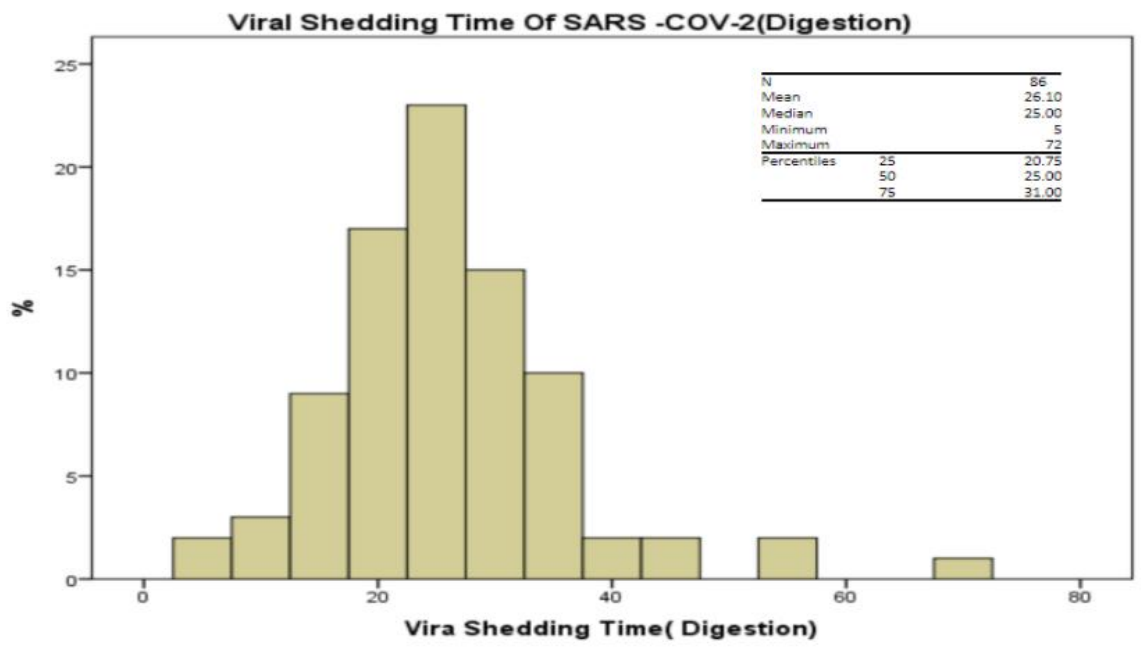

B

Figure 1: diagram of novel coronaviruses in the digestive and respiratory tract viral clearance time, with digestive tract specimens being faeces, respiratory specimens being sputum or nasopharyngeal swabs or throat swabs, and most specimens being sputum specimens. 


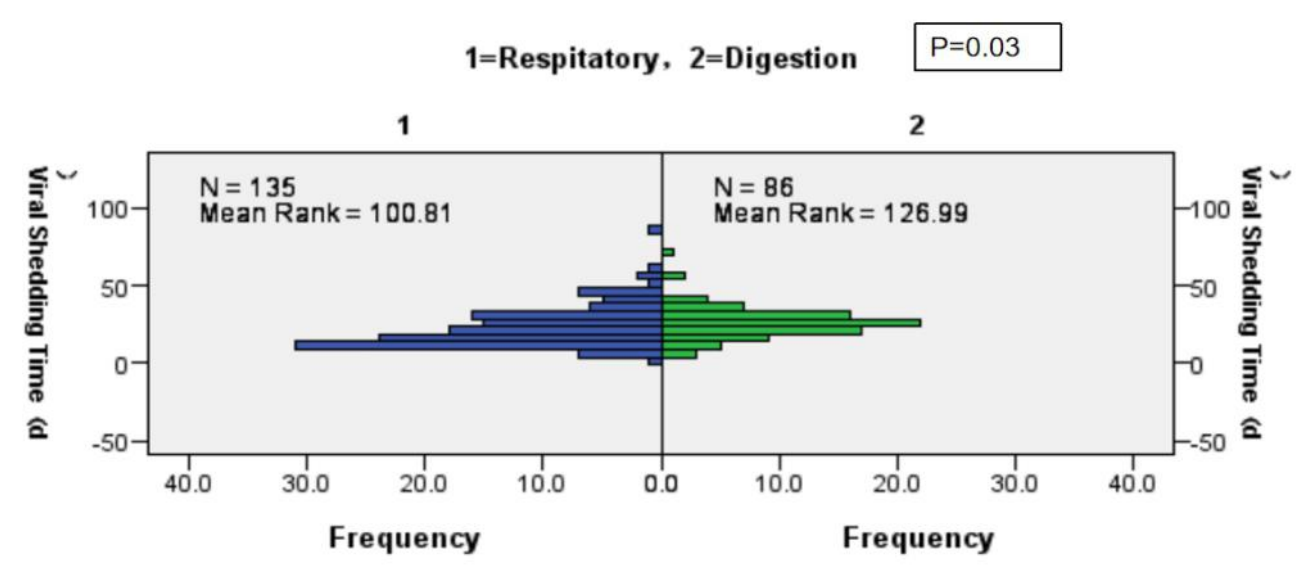

Figure3 : The comparison of Viral Shedding/Clearance Time of SARS-COV-2 in Digiestion and Respitory

In our analysis, we found the clearance time of SARS-CoV-2 to vary considerably, with some patients having maximum respiratory clearance times of as high as 173 days. For ease of statistical analysis, we excluded the outliers with viral clearance times greater than 100 days and analyzed the data of the remaining 135 patients. Among these included patients, the average time for virus clearance in the respiratory tract was 22.84 days; the median time was $20(13,30)$ days, the maximum time was 84 days, and the minimum time was 1 day. In a simultaneous fecal test to identify the disease etiology, the viral RNA was detected in 86 patients. The findings indicated that the virus was cleared from the digestive tract after a mean duration of 26.1 days, with a median clearance time of $25(20.75,31)$ days, with a minimum of 5 days and a maximum of 72 days, relative to the viral strain that was cleared from the digestive tract after a longer duration than that from the respiratory system $(P=0.03$, Figure 2$)$.

\section{Risk factors for prolonged SARS-CoV-2 RNA shedding}

We collected data related to routine blood analysis, biochemical evaluations, and TBNK, lymphokines, and blood gas analysis of patients in the early stage of the disease as well as the follow-up results of the antibody test. Moreover, the clinical manifestations and presentation during the remission of symptoms were also considered to analyze the factors related to the clearance time of SARS-CoV-2. The correlation between disease severity and antibody production, advanced age, 
presence of comorbidities, the use of hormonal therapy, immune-function deficiency, and virus clearance time was analyzed.

Table 2. Rank correlation analyses of factors associated with duration of SARS-CoV-2 Virus RNA detection

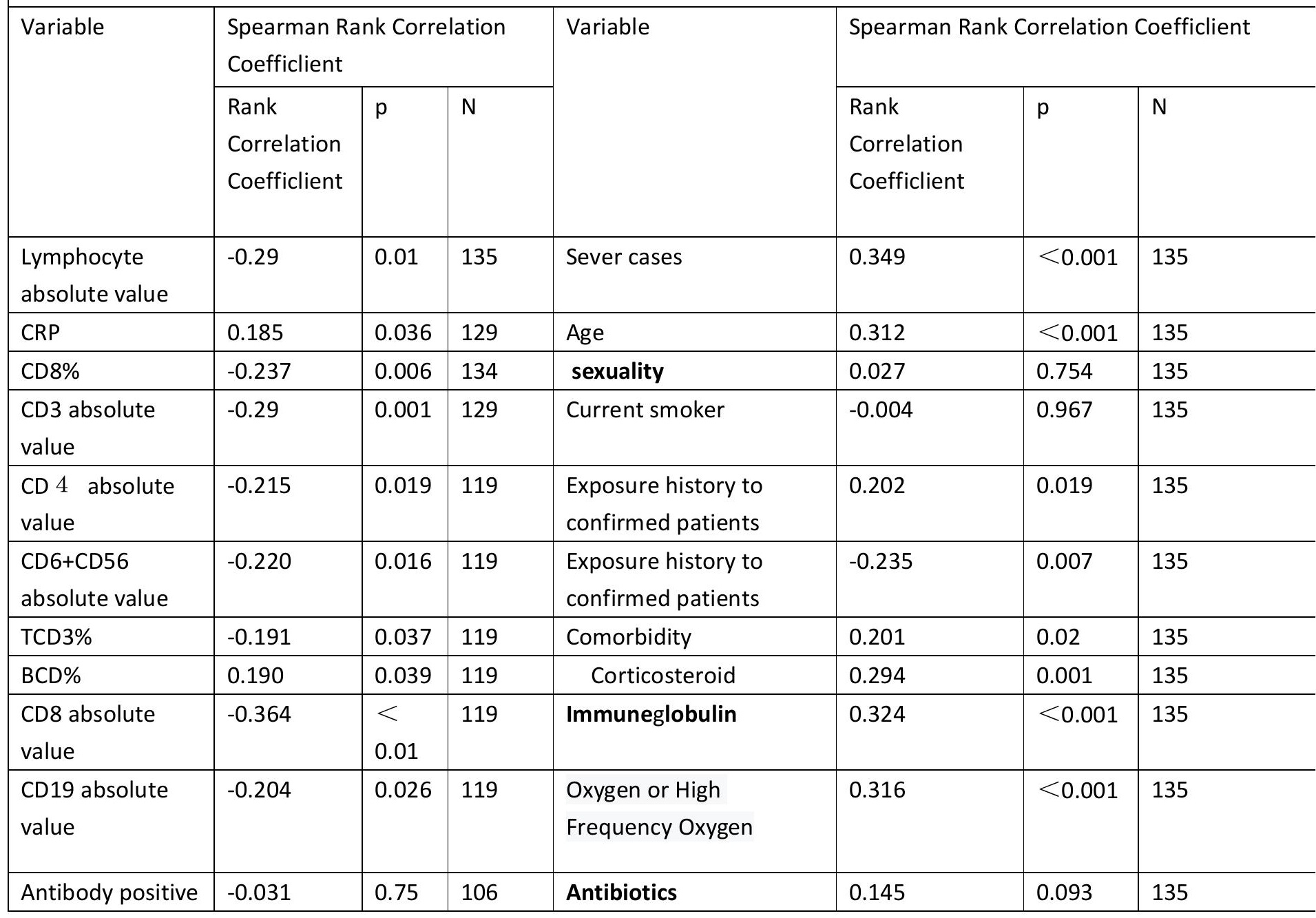




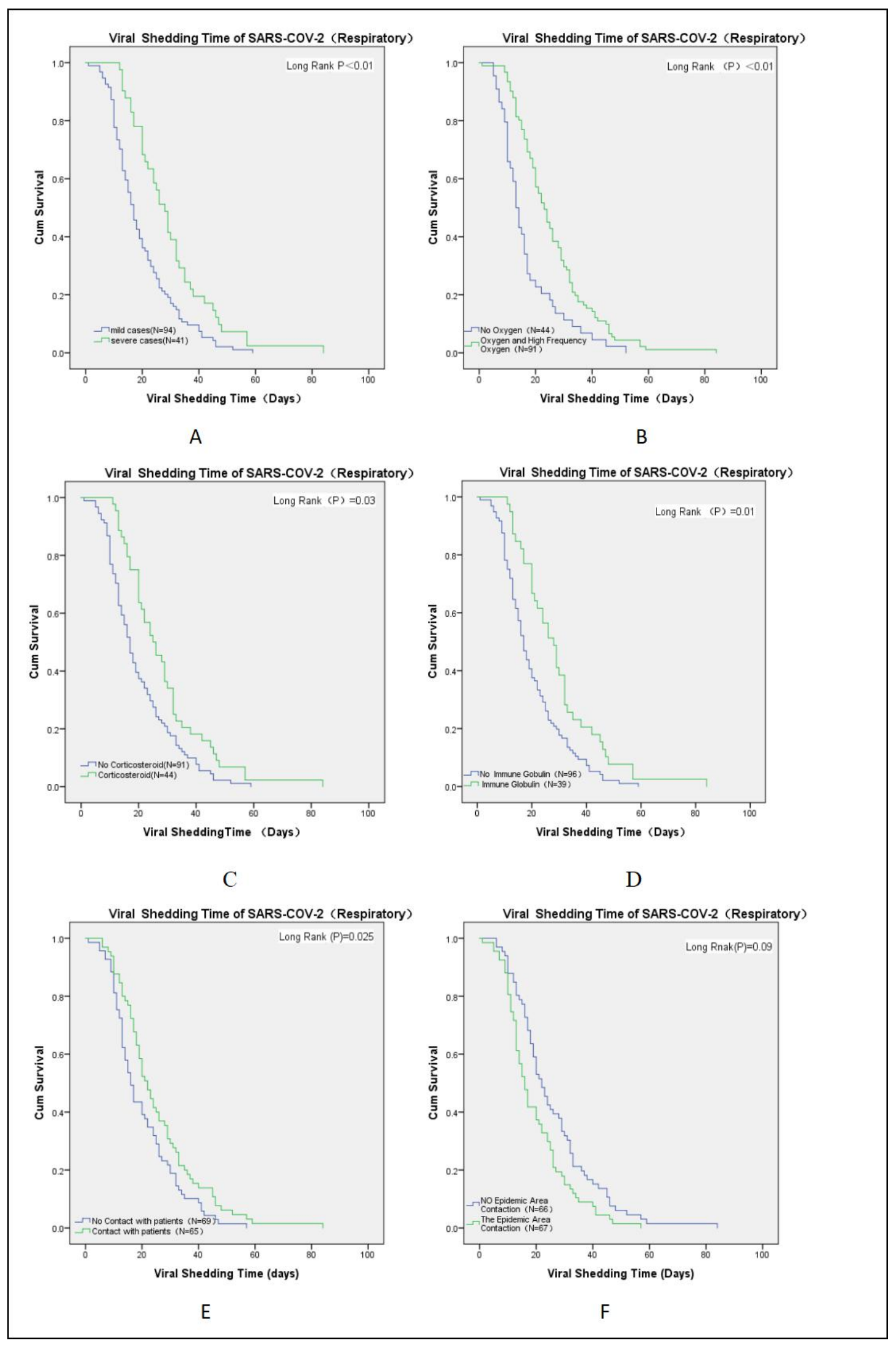




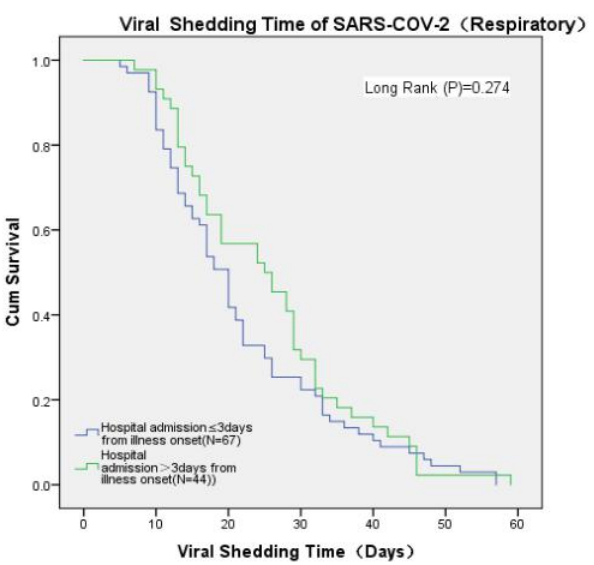

G

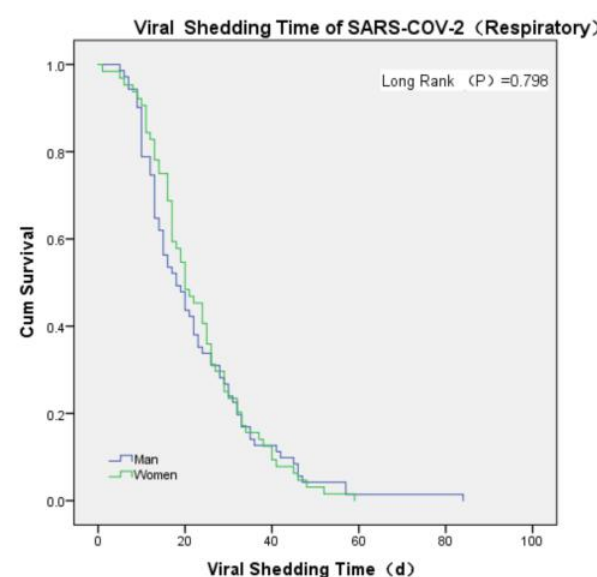

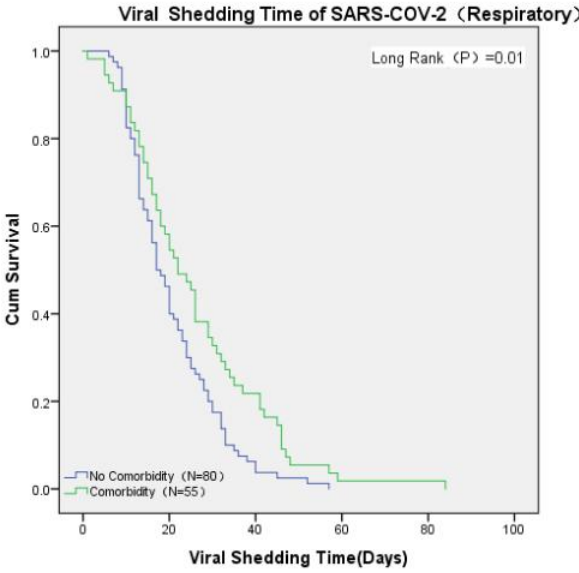

$\mathrm{H}$

Figure4: A. Viral Shedding Time of SARS-COV-2 ( Respiratory) between mild cases and sever case (log-rank P $<0.01$ ). B.Viral Shedding Time of SARS-COV-2 ( Respiratory) between patients whether use oxygen or high frequency Oxygen(log-rank P <0.01) ; C.Viral Shedding Time of SARS-COV-2 ( Respiratory) between patients whether use corticosteroid (log-rank $P=0.03$ ). D. Viral Shedding Time of SARS-COV-2 ( Respiratory) between patients whether use immuneglobulin (log-rank $P=0.01)$. E.Viral Shedding Time of SARS-COV-2 ( Respiratory ) between patients whether contact to confirmed patients(log-rank $P=0.025)$; F. Viral Shedding Time of SARS-COV-2 ( Respiratory ) between patients whether exposure in Epidemic Area (log-rank P =0.09). G.Viral Shedding Time of SARS-COV-2 ( Respiratory) between patients' duration from illness onset to hospital admission less than 3days and more than 3days(log-rank $\mathrm{P}=0.274)$;H.Viral Shedding Time of SARS-COV-2 ( Respiratory ) between patients whether have comorbidity (log-rank $P=0.01$ ).I.Viral Shedding Time of SARS-COV-2 ( Respiratory ) between man and woman (log-rank $\mathrm{P}=0.798$ )

Based on the results of our study, we found that the time to clearance of SARS-CoV-2 from the respiratory system was significantly (Figure 4, A, log-rank $P<$ 0.01 ) associated with the use of hormones (Figure $4, C$, log-rank $P=0.03$ ), the use of immunoglobulins (Figure 4, D, log-rank $p=0.01$ ), the use of oxygen therapy (Figure3, $B, \log$-rank $P<0.01$ ), history of exposure of affected patients (Figure $4, E$, log-rank $P$ $=0.025$ ), and the presence of comorbidities (Figure 4, H, log-rank P = 0.09); that is, patients who were critically ill and received hormone therapy, immunosuppressive agents, or oxygen therapy, and those with comorbidities were found to exhibit 
prolonged clearance of the novel coronavirus. Interestingly, we found that prolonged clearance of the novel coronavirus was associated with patients with a history of visits to areas where the epidemic was prevalent. (Figure 4, F, log-rank $P=0.09$ ). Additionally, patients who had a history of contact with individuals in epidemic-prone areas showed a longer virus clearance time than those without a history of contact. Moreover, the time from disease onset to hospital admission (Figure 4, G, log-rank $=0.274$ ) and gender (Figure 4 , I, log-rank $=0.798$ ) were not significantly different in terms of respiratory clearance time of the virus.

To further test our hypothesis, we performed a rank correlation analysis to evaluate the results of laboratory tests, including blood routine, biochemical, immunological series, new corona antibodies, and blood gas analysis, in relation to the clearance time of SARS-CoV-2. The results are shown in Table 2.

Our findings indicated that the main factors affecting viral clearance time in the respiratory tract are the lymphocyte counts, including T cell (absolute CD3, CD4, and CD8 levels) and B cell (absolute CD 19 counts) immunity, and NK cell (CD16 + CD56 absolute values) immunity. High levels of lymphocytes were negatively correlated with the novel coronavirus virus clearance time, i.e., lymphopenia, with a prolonged clearance time of the novel coronavirus, which is consistent with our findings that in immunocompromised patients, the SARS-CoV-2 clearance time would be prolonged. Additionally, we found that CRP levels were positively correlated with the virus clearance time in the respiratory tract (Table $2, \mathrm{P}=0.036)$. In other words, the higher the CRP, the longer the virus clearance time. The presence of antibodies had little relationship with the respiratory clearance time of patients $(P=0.75)$. Among other factors, we found that critically ill patients, the use of hormones and immunoglobulins, oxygen therapy, advanced age, and patients with comorbidities were positively correlated with SARS-CoV-2 clearance time, which was consistent with data from our previous analysis. Importantly, smoking, gender, and the use of antibiotics did not play a role in the clearance time of SARS-CoV-2.

\section{Discussion:}

Current studies on COVID-19 are generally limited to the description of early clinical, hematological, and radiological findings. To date, only a few studies have reported the time required for RNA shedding of SARS-CoV-2, and among these, the 
data are mostly limited to the early stages of the outbreak. Ours is the first study to follow up on patients with COVID-19 and to determine whether they were completely negative for the antibody after a period of 8 months. In this study, we also documented the risk factors associated with complete clearance of SARS-CoV-2 in the respiratory tract.

We determined that the median clearance time of SARS-CoV-2 from the respiratory tract was $20(13,30)$ days, with a mean clearance time of 22.84 (Figure 2, A). The median clearance time of SARS-CoV-2 from the digestive tract was $25(20.75$, 31) days, with a mean clearance time of 26.1 (Figure 2, B). The viral shedding time of SARS in the digestive tract was determined to be longer than that in the respiratory tract $(p=0.03$, Figure 3$)$.

Viral clearance was found to be a maximum of 173 days in our study and five patients exhibited viral clearance time greater than 100 days (3.6\%). For ease of statistical analysis, we excluded these extreme values. Chenliang Zhou et al. reported the viral clearance time of 111 days in a male patient; the median viral clearance time of patients was determined to be 32 days in that study. Most patients developed viral shedding within 60 days[9] in contrast to that determined in our study as well as a study with 113 patients with COVID-19 that reports the median duration of viral RNA as 17 days[10].

Our findings indicated that the viral clearance from the digestive system was longer than that observed for the respiratory system (Figure 3, P = 0.03). There exists the possibility of transmission via the fecal or oral route in patients who test negative for novel coronavirus RNA in sputum samples[8,11]. Even if negative test results are consistent when multiple sputum specimens are analyzed, repeated analysis of stool specimens do not consistently yield a negative antibody result, which indicates a greater likelihood of reinfection at a later stage. Therefore, the management of excretions of patients diagnosed with COVID-19 needs to be addressed and further enhanced $[2,5]$.

We found that disease severity, the use of hormones and immunoglobulins, oxygen therapy, advanced age, and comorbidity were associated with prolonged 
clearance time of SARS-CoV-2; however, gender, and the administration of antibiotics did not affect viral clearance time (Figure 4, Table 2), unlike the study in which Kaijin Xu et al. concluded that male sex was an independent risk factor associated with prolongation of viral clearance[10]. In addition, Chenliang Zhou et al. reported that disease severity was not significantly associated with viral clearance time; however, age was positively associated with viral clearance time[9]. As therapy involving hormones, immunoglobulins, and high-frequency oxygen inhalation was reserved only for critically ill patients, these factors do not serve as independent risk factors for prolonged clearance of the novel coronavirus.Moreover,we did not have special intervention for patients with comorbidities or elderly patients; therefore, we have reason to suggest that severe disease and advanced age may serve as independent risk factors in this aspect.This may be owing to the combination of important basic diseases and advanced age,which weaken the ability of the patient to resist infections, thereby prolonging the shedding time of SARS-COV-2.

Patients who had direct contact with an infected individual had prolonged viral shedding times. On the other hand, patients who developed the infection after visiting an epidemic-stricken area had a comparatively shorter viral shedding time compared to those who did not visit areas affected by the epidemic. This may be associated with less airborne viral loads; however, the specific details need to be further studied.

In terms of laboratory tests, we found that lymphopenia, including low levels of T, B, and NK lymphocytes, was an independent risk factor contributing to prolonged viral clearance (Table 2, $\mathrm{P}=0.01)[12]$. Laboratory tests were suggestive of lymphopenia as a common presentation. In a recent study, lymphopenia was observed in 914 (83.2\%) of the 1099 enrolled patients[13].Another study showed that patients with severe COVID-19 had significantly fewer T cells (especially CD8+ T cells) compared to those with the mild form of the disease[14]. The expression of PD-1 in T cells of COVID-19 patients was significantly higher compared to that in the healthy controls[12]. Patients with COVID-19 have decreased CD3+, CD4+, CD8+ T cells, CD19+ B cells, and CD16+/CD56+ NK cells in the blood[15], which can be 
attributed to disease severity. Furthermore, lymphopenia may be a risk factor associated with cytokine storms and is likely a major cause affecting the clearance of SARS-CoV-2. In our study, CRP levels were positively correlated with viral clearance time (Table 2, P = 0.036). CRP is rapidly synthesized when hepatocytes are stimulated during inflammation. CRP binds to several eukaryotic and prokaryotic pathogens and promotes complement activation via the classical pathway, suggesting immune activation, lymphocytic infiltration, depletion of immune molecules, and cytokine storm. In a clinical setting, elevated CRP levels serve as an early indicator of nosocomial infections in patients with COVID-19. These patients take longer to recover; therefore, early commencement of empirical antibiotic therapy may prevent deterioration[16]. We analyzed the data of 106 patients and found that 93 patients were positive for SARS-CoV-2 antibodies, $87.7 \%$ were negative, and 93 patients were persistently negative at the 8-month follow-up. Thus, the presence of antibodies did not correlate with the clearance time of SARS-CoV-2 (Table 2, P = 0.75). Additionally, two patients tested positive for COVID-19 at the follow-up, although the disease intensity diminished at follow-up compared to the initial findings. Whether the SARS-CoV-2 antibody has a protective effect on patients warrants further studies.

\section{Summary}

SARS-COV-2 took longer to clear from the digestive tract compared to that in the respiratory tract. Disease severity, advanced age, elevated CRP levels, and lymphopenia were independent risk factors for prolonged viral clearance, whereas gender and the presence of antibodies did not affect the clearance time of this respiratory virus. In the current study,we determined the virus shedding time and factors influencing clearance based on a follow-up of patients for up to 8 months.Compared to that of previous studies, the conclusion deriverd from this present study are more convincing.Additionally, we also confirmed that low immunity is the primary reson leading to the prolonged clearance time of SARS-COV-2.This aspect is of great significance for predicting patient isolation time and guiding the next steps in preventing the spread of the epidemic. However, our study has some shortcomings. First, our data is relatively limited and comprised only 135 cases. 
Second, because of the discrepancy in findings compared to that of published studies, a larger patient pool is required to further strengthen our findings. Lastly,our study only compares and evaluates the initial laboratory datas, which represents the initial test status of the patient.It does not analyze the subsequent dynamic observation laboratory datas. However, in this study,we found that the disease severity of some patients have changed from mild to severe.It is,therefore,important to monitor these patients over time.

\section{Abbreviation:}

Coronavirus disease 2019 (COVID-19), Severe acute respiratory syndrome coronavirus 2 (SARS-CoV-2), Acute respiratory distress syndrome (ARDS), Interquartile range (IQR), Real-time reverse transcription-polymerase chain reaction (RT-PCR), Middle East Respiratory Syndrome (MERS)

\section{Declarations}

\section{Acknowledgements}

This work was funded by The Science and Technology Project of Taizhou(1902kynology).We thank the CDCs of Taizhou City and zhejian Province for helping with confirmation of the diagnosis of the viral infection.

\section{Author Contributions}

DQL contributed to the study design and data interpretation. GXW contributed to data analysis, data interpretation and writing of the manuscript. GXW , XMW,QC,SSH,YPJ, LL and SQY contributed to clinical management, patients' data collection. LL,WJP contributed to critical revision of the manuscript. All authors reviewed and approved the final version of the manuscript.

\section{Funding}


This work was supported by The Science and Technology Project of Taizhou(no.1902ky27)

\section{Availability of data and materials}

All data generated or analyzed are included in this published article.

\section{Ethics approval and consent to participate}

Ethical approval was granted by the Ethics Committee /Institutional Review board of Enze Hospital of Taizhou ENze Medical (CenterNO.K20200204).Informed consent was obtained from patients or their family members.

\section{Consent for publication}

Not applicable

\section{Competing interests}

No conflict of interest to declare.

\section{Author details}

${ }^{1}$ Taizhou Hospital of Zhejiang Province,Zhejian University Enze Hospital,Taizhou Enze Mesical Center(Group) ,Department of Respiratory and Critical Care Medicine,Taizhou ,Zhejiang Province,China,318050

Guixian-Wu,Ling-Lin,Qian-Chen,Xiaomai-Wu,Shuangquan-Yan,Weijia-Pan,Don gqin-Lv

${ }^{2}$ Taizhou Hospital of Zhejiang Province,Zhejian University Department of Respiratory and Critical Care Medicine,Taizhou ,Zhejiang Province, China,317000

Susu-He

${ }^{3}$ Taizhou Hospital of Zhejiang Province,Zhejian University,Taizhou ,Zhejiang Province,ICU, Taizhou ,Zhejiang Province, 317000

Yongpo Jiang

\section{References}

1. Jiang, F., et al. Review of the Clinical Characteristics of Coronavirus Disease 2019 (COVID-19). J Gen Intern Med, 2020.

2. Foladori, P., et al., SARS-CoV-2 from faeces to wastewater treatment: 
What do we know? A review. Sci Total Environ, 2020. 743: p. 140444.

3. Alviset, S., et al., Continuous Positive Airway Pressure (CPAP) face-mask ventilation is an easy and cheap option to manage a massive influx of patients presenting acute respiratory failure during the SARS-CoV-2 outbreak: A retrospective cohort study. PLoS One, 2020. 15(10): p. e0240645.

4. Gupta, S., et al., Persistent viral shedding of SARS-CoV-2 in faeces - a rapid review. Colorectal Dis, 2020. 22(6): p. 611-620.

5. Quilliam, R.S., et al., COVID-19: The environmental implications of shedding SARS-CoV-2 in human faeces. Environ Int, 2020. 140: p. 105790.

6. Jiang, F., et al., Review of the Clinical Characteristics of Coronavirus Disease 2019 (COVID-19). J Gen Intern Med, 2020. 35(5): p. 1545-1549.

7. Li, Y., et al., Positive result of Sars-Cov-2 in faeces and sputum from discharged patients with COVID-19 in Yiwu, China. J Med Virol, 2020.

8. Han, H., et al., SARS-CoV-2 RNA more readily detected in induced sputum than in throat swabs of convalescent COVID-19 patients. Lancet Infect Dis, 2020. 20(6): p. 655-656.

9. Zhou, C., et al., Impact of age on duration of viral RNA shedding in patients with COVID-19. Aging (Albany NY), 2020. 12(22): p. 22399-22404.

10. $\mathrm{Xu}, \mathrm{K}$. , et al., Factors Associated With Prolonged Viral RNA Shedding in Patients with Coronavirus Disease 2019 (COVID-19). Clin Infect Dis, 2020. 71(15): p. 799-806.

11. Zhang, W., et al., Molecular and serological investigation of 2019-nCoV infected patients: implication of multiple shedding routes. Emerg Microbes Infect, 2020. 9(1): p. 386-389.

12. Zhou, G., S. Chen, and Z. Chen, Advances in COVID-19: the virus, the pathogenesis, and evidence-based control and therapeutic strategies. Front Med, 2020. 14(2): p. 117-125.

13. Guan, W.J., et al., Clinical Characteristics of Coronavirus Disease 2019 in China. N Engl J Med, 2020. 382(18): p. 1708-1720.

14. Liu, J., et al., Longitudinal characteristics of lymphocyte responses and cytokine profiles in the peripheral blood of SARS-CoV-2 infected patients. EBioMedicine, 2020. 55: p. 102763.

15. Zhang, Z., et al., Clinical Features and Treatment of 2019-nCov Pneumonia Patients in Wuhan: Report of A Couple Cases. Virol Sin, 2020. 35(3): p. 330-336.

16. Chen, W., et al., Plasma CRP level is positively associated with the severity of COVID-19. Ann Clin Microbiol Antimicrob, 2020. 19(1): p. 18. 
Figures

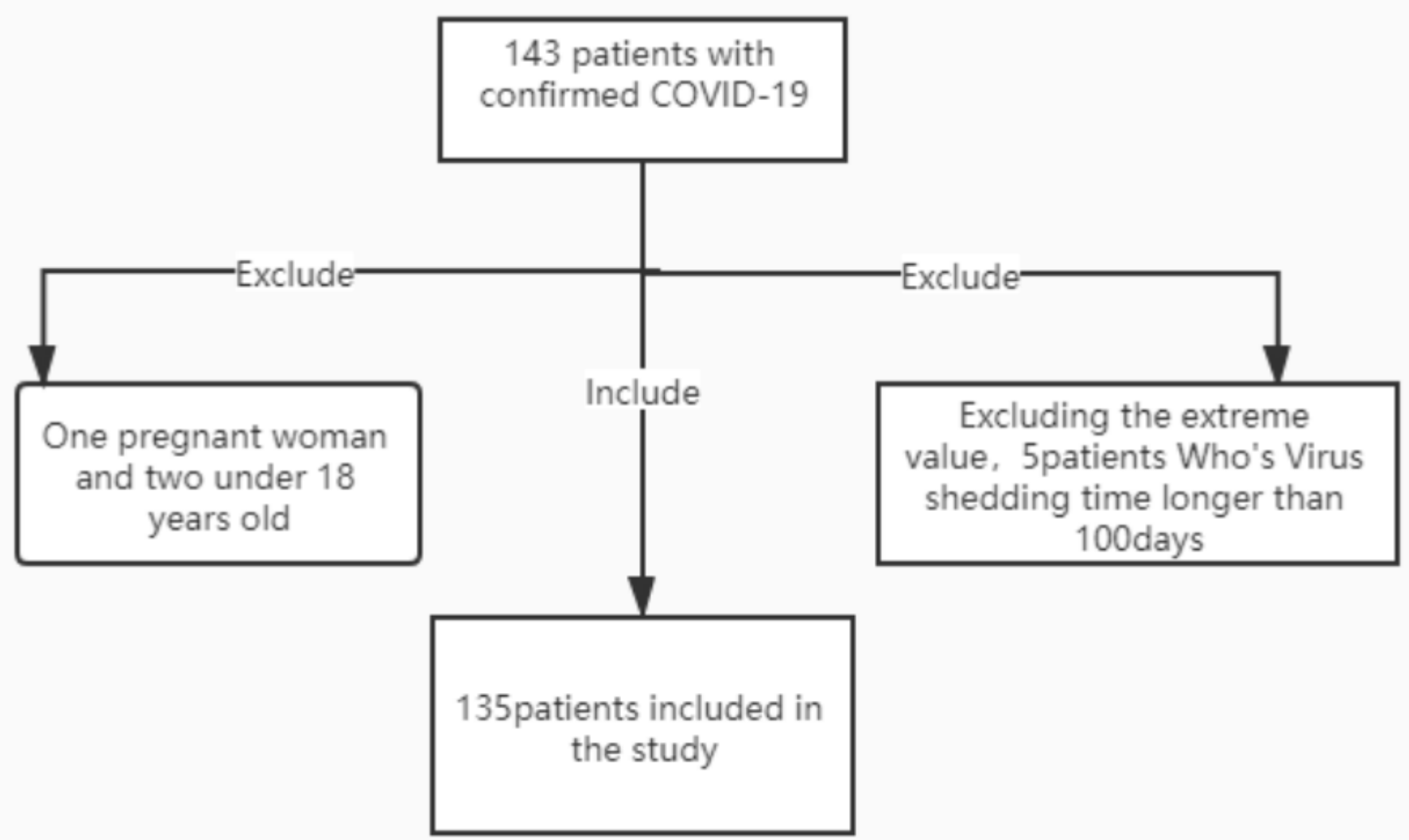

\section{Figure 1}

Flow diagram of patients with confirmed COVID-19 included in this study 


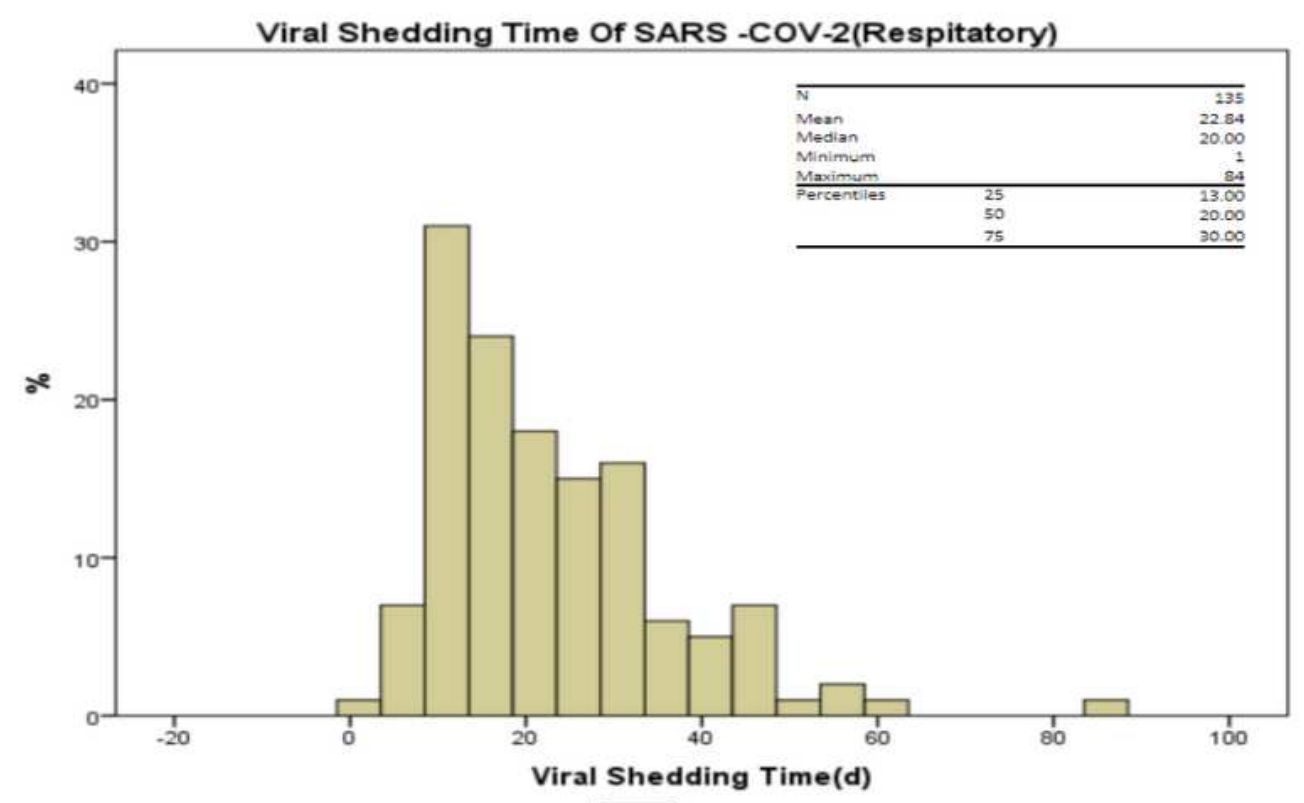

A

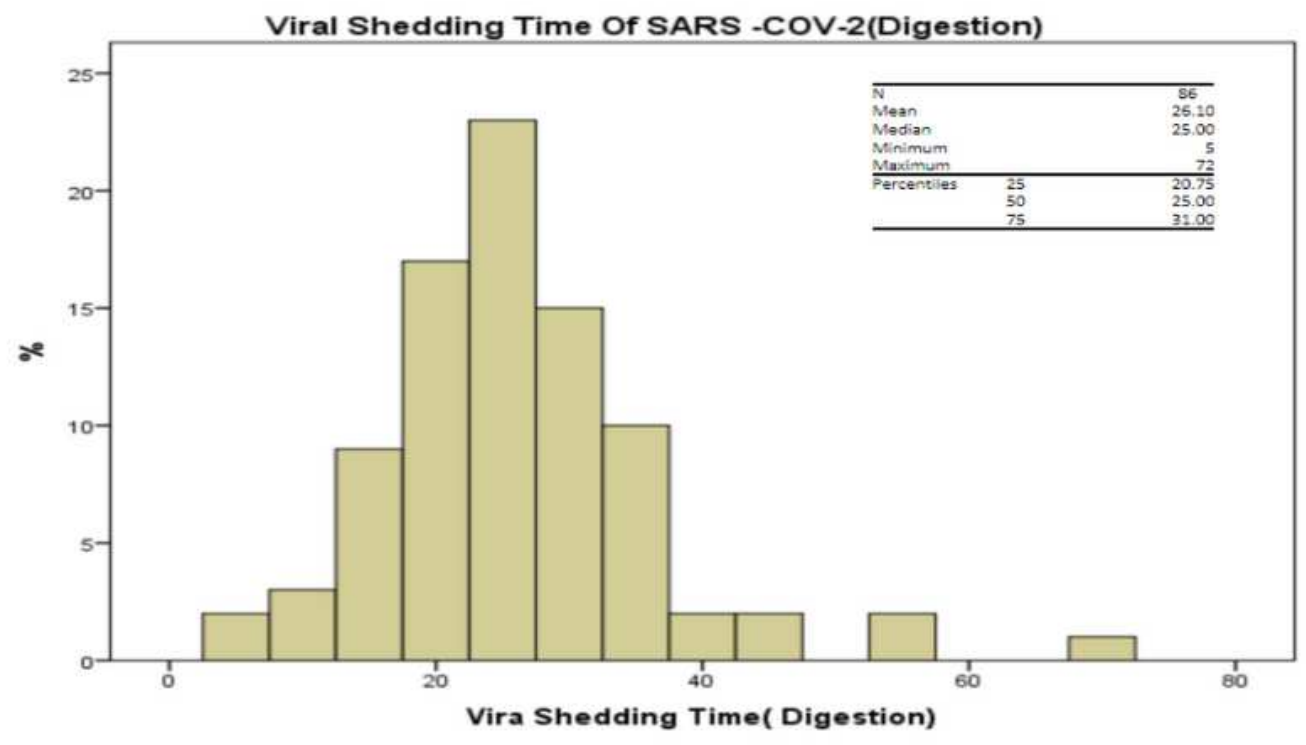

B

\section{Figure 2}

Viral Sheddin / Clearance Time of SARS-COV-2 in Digestion and Respitory diagram of novel coronaviruses in the digestive and respiratory tract viral clearance time, with digestive tract specimens being faeces, respiratory specimens being sputum or nasopharyngeal swabs or throat swabs, and most specimens being sputum specnnens. 


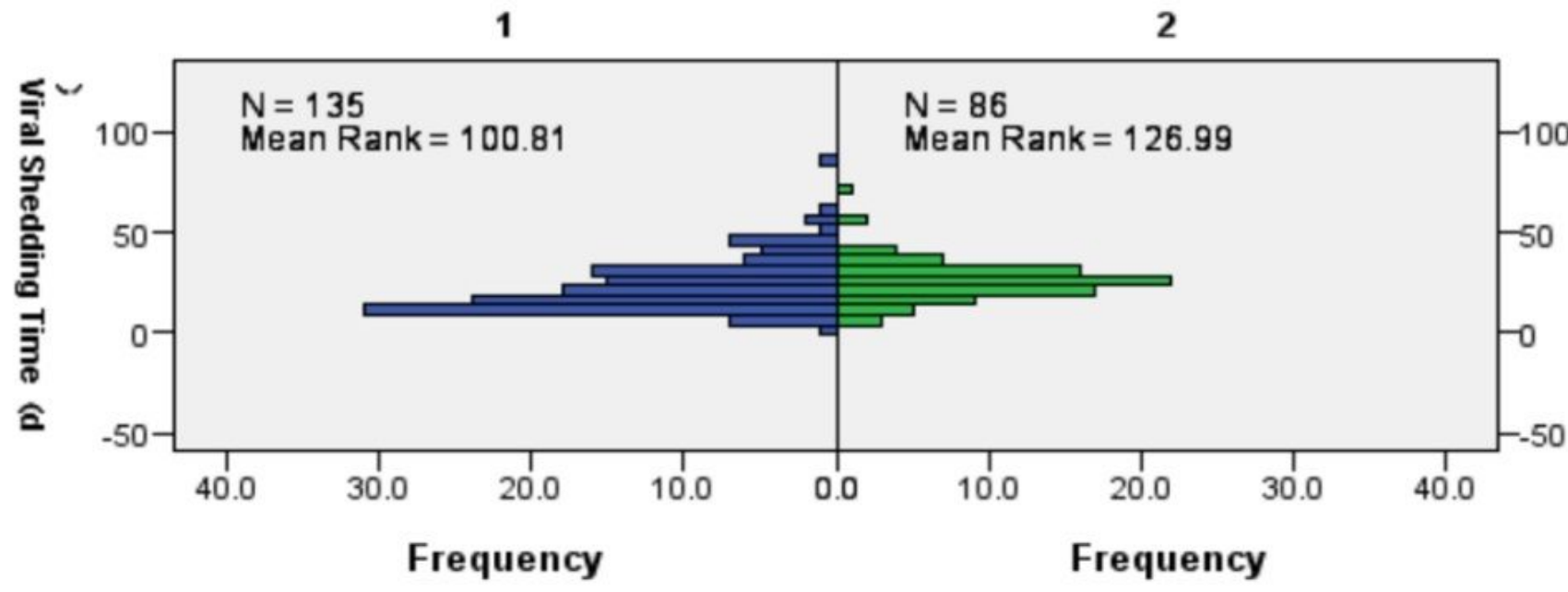

Figure 3

The comparison of Viral Shedding / Clearance Time of SARS-COV-2 in digestion and respiratory

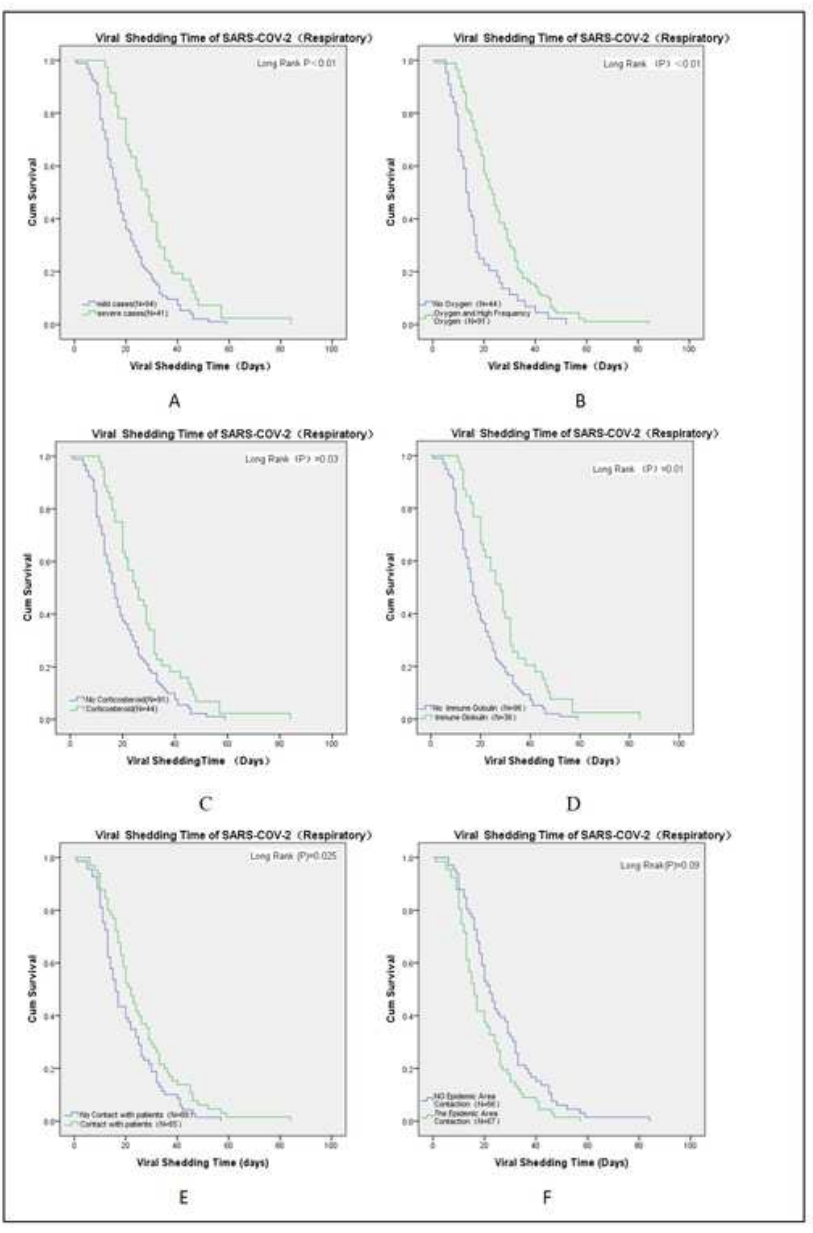

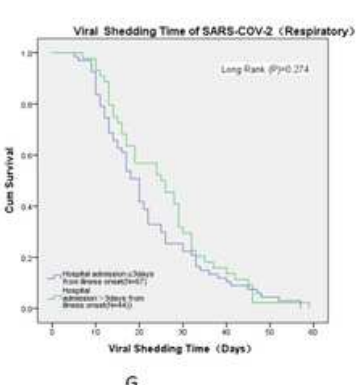

G

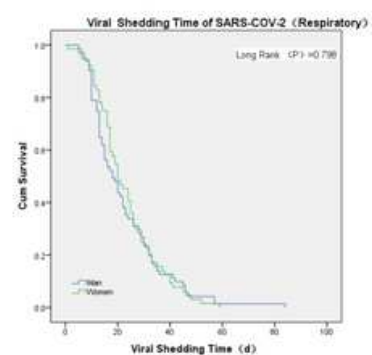

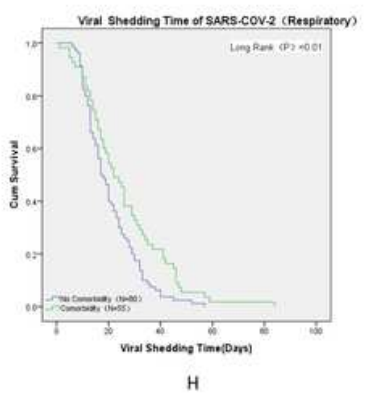

$\mathrm{H}$ 
Figure 4

Please see the Manuscript PDF file for the complete figure caption. 